\title{
New results on slowly varying functions in the Zygmund sense
}

\author{
By Edward OMEY*) and Meitner CADENA*) \\ (Communicated by Masaki KashiwarA, M.J.A., June 12, 2020)
}

\begin{abstract}
Very recently Seneta [15] has provided a characterization of slowly varying functions $L$ in the Zygmund sense by using the condition, for each $y>0$,

$$
x\left(\frac{L(x+y)}{L(x)}-1\right) \rightarrow 0 \text { as } x \rightarrow \infty .
$$

We extend this result by considering a wider class of functions and a more general condition than (1). Further, a representation theorem for this wider class is provided.

Key words: Slowly varying; monotony in the Zygmund sense; class $\Gamma_{a}(g)$; self-neglecting function; extreme value theory; convergence rates.
\end{abstract}

1. Introduction. The notion of ultimately monotony introduced by Zygmund dates back to works like [16, p. 237] and [17, p. 186]. It says that a function $U \geq 0$ is slowly varying if for each $\epsilon>0$ the function $x^{\epsilon} U(x)$ is ultimately increasing and $x^{-\epsilon} U(x)$ is ultimately decreasing. The class of this type of functions is called the Zygmund class (ZSV). A different kind of slowly varying functions was defined by Karamata [6,7] known as simply the class of slowly varying functions (KSV). It is known that any ZSV function is a KSV function, see [17, p. 186] and e.g. [14, p. 49].

A number of authors have analyzed generalizations of the functions ZSV, by considering $U(x)$ / $x^{a}$ is increasing and $U(x) / x^{b}$ is decreasing, with $-\infty<a \leq b<\infty[3,4,11]$. This type of functions have applications in analysis, differential equations and approximation theory $[4,5,9,12]$. Also, they are related with the notion of quasi-convexity, leading to applications in probability $[8,13]$.

Very recently [15] has given an elegant characterization of the ZSV functions in terms of the condition (1).

In this paper, we extend this result by considering a wider notion of ultimately monotony than that proposed by Zygmund. Consequently, a more general limit than that involved in (1) is provided. To this aim, we take into account functions related to self-neglecting functions and to functions belong-

2010 Mathematics Subject Classification. Primary 26A12, 28A10, 45M05, 60G70.

*) KU Leuven, Campus Brussels, Dept. MEES, Warmoesberg 26, 1000 Brussels, Belgium.

**) DECE, Universidad de las Fuerzas Armadas, Ecuador. ing to $\Gamma_{0}(g)$. This type of functions have been deeply studied in [10].

In what follows, a brief review of functions that belong to the gamma class and related classes of functions is shown. Next, the new class called $Z(g, a)$ is introduced and then a result of Zygmund type. That section then presents an extension of Theorem 2 in [15] and a characterization of the members of $Z(g, a)$.

\section{Main Results.}

2.1. Preliminaires. In Omey [10] the author studied the following class of functions.

The positive and measurable function $g$ is selfneglecting (notation: $g \in S N$ ) if it satisfies

$$
\frac{g(x+y g(x))}{g(x)} \rightarrow 1, \forall y \in \mathbf{R}
$$

and locally uniformy in $y$.

The positive and measurable function $a$ is in the class $\Gamma_{\alpha}(g)$ if $g \in S N$ and if

$$
\frac{a(x+y g(x))}{a(x)} \rightarrow e^{\alpha y}, \forall y \in \mathbf{R} .
$$

In [10] it is proved that this relation automatically holds locally uniformly in $y$.

The positive and measurable function is in the class $E \Gamma_{\alpha}(g, a)$ if $g \in S N, a \in \Gamma_{0}(g)$ and

$$
\frac{f(x+y g(x))-f(x)}{a(x)} \rightarrow \alpha y, \forall y \in \mathbf{R} .
$$

In [10] it is proved that this relation automatically holds locally uniformly in $y$.

Note that if $a(x) / f(x) \rightarrow 0$, we find that $f \in$ $\Gamma_{0}(g)$ with a remainder term. 
In [10] the author proved that for $g \in S N$ and $a \in \Gamma_{0}(g)$ we have $f \in E \Gamma_{\alpha}(g, a)$ if and only if $f$ is of the form $f(x)=A(G(x))$, where $A \in \Pi_{\alpha}(L)$ and $G \in$ $\Gamma_{1}(g)$ where $L \in R V_{0}$ and $g \in S N$.

In [10, Theorem 2.2] the author also showed that for $g \in S N$ and $a \in \Gamma_{0}(g)$ we have $f \in E \Gamma_{\alpha}(g, a)$ if and only if $f$ can be represented as

$$
f(x)=C+W(x)+\alpha \int_{x^{\circ}}^{x} \frac{a(t)}{g(t)} d t+V(x),
$$

where $V(x) / a(x) \rightarrow 0$ and $W^{\prime}(x) g(x) / a(x) \rightarrow 0$.

2.2. The class $Z(g, a)$. In view of Zygmund we consider the following class of functions. We assume that $g \in S N$ and $a \in \Gamma_{0}(g)$ and we also assume that $g(x)=o(a(x))$. The positive and measurable function $U(x)$ is in the class $Z(g, a)$ if it satisfies:

(2) $\lim _{x \rightarrow \infty} \frac{a(x)}{g(x)}\left(\frac{U(x+y g(x))}{U(x)}-1\right)=0, \forall y \in \mathbf{R}$.

From (2) it follows that $U \in \Gamma_{0}(g)$ and also that

(3) $\lim _{x \rightarrow \infty} \frac{a(x)}{g(x) U(x)}(U(x+y g(x)-U(x))=0, \forall y \in \mathbf{R}$,

so that $U \in E \Gamma_{0}(g, b)$, where $b(x)=g(x) U(x) /$ $a(x) \in \Gamma_{0}(g)$.

From (1) it also follows that

$$
\lim _{x \rightarrow \infty} \frac{a(x)}{g(x)} \log \frac{U(x+y g(x))}{U(x)}=0, \forall y \in \mathbf{R},
$$

so that $\log U(x) \in E \Gamma_{0}(g, b)$ where $b(x)=g(x) /$ $a(x) \in \Gamma_{0}(g)$.

From the previous subsection we have that in each of the relations (2)-(4), the relation holds l.u. in $y \in \mathbf{R}$.

The representation result of the previous subsection (with $\alpha=0$ ) shows that $\log U(x)$ can be written as:

$$
\log U(x)=C+W(x)+V(x),
$$

where $V(x) a(x) / g(x) \rightarrow 0$ and $W^{\prime}(x) a(x) \rightarrow 0$.

2.3. Result of Zygmund type. A positive and measurable function $L$ is in the Zygmund class of slowly varying functions if for any fixed $\epsilon>0$ and any $y>0$,

$$
x^{\epsilon} L(x) \leq(x+y)^{\epsilon} L(x+y)
$$

and

$$
x^{-\epsilon} L(x) \geq(x+y)^{-\epsilon} L(x+y) .
$$

This class of functions is a subclass of the class of slowly varying functions in the sense of Karamata.

Seneta [15] proved that $L$ is in the Zygmund class of SV functions if and only if

$$
\lim _{x \rightarrow \infty} x\left(\frac{L(x+y)}{L(x)}-1\right)=0, \forall y \in \mathbf{R} .
$$

In this section we generalize the result of Seneta to the class $Z(g, a)$. We prove the following result:

Theorem 1. Assume that $g \in S N, a \in \Gamma_{0}(g)$ and $g(x)=o(a(x))$. Let $y>0$. The inequalities, for $x$ large and $\epsilon>0$,

$$
\begin{gathered}
U(x+y g(x)) \times \exp \epsilon \int_{x^{\circ}}^{x+y g(x)} a^{-1}(t) d t \\
\geq U(x) \times \exp \epsilon \int_{x^{\circ}}^{x} a^{-1}(t) d t
\end{gathered}
$$

and

$$
\begin{gathered}
U(x+y g(x)) \times \exp -\epsilon \int_{x^{\circ}}^{x+y g(x)} a^{-1}(t) d t \\
\leq U(x) \times \exp -\epsilon \int_{x^{\circ}}^{x} a^{-1}(t) d t,
\end{gathered}
$$

hold if and only if $U \in Z(g, a)$.

Proof. Assume that $U \in Z(g, a)$. Let $y>0$. We consider for $\epsilon \in \mathbf{R}$ the function $V(x)=U(x) \times$ $\exp \epsilon \int_{x^{\circ}}^{x} a^{-1}(t) d t$.

We then have

$$
\begin{aligned}
& \frac{V(x+y g(x))-V(x)}{V(x)} \\
& \quad=\frac{U(x+y g(x))}{U(x)} \exp \epsilon \int_{x}^{x+y g(x)} a^{-1}(t) d t-1 \\
& =I+I I,
\end{aligned}
$$

where

$$
\begin{aligned}
I & =\left(\frac{U(x+y g(x))}{U(x)}-1\right) \exp \epsilon \int_{x}^{x+y g(x)} a^{-1}(t) d t, \\
I I & =\exp \epsilon \int_{x}^{x+y g(x)} a^{-1}(t) d t-1 .
\end{aligned}
$$

In the second term we have (put $t=x+\theta g(x)$ )

$$
\int_{x}^{x+y g(x)} a^{-1}(t) d t=\frac{g(x)}{a(x)} \int_{0}^{y} \frac{a(x)}{a(x+\theta g(x))} d \theta .
$$

Since $a \in \Gamma_{0}(g)$, we have

$$
\int_{x}^{x+y g(x)} a^{-1}(t) d t \sim \frac{g(x)}{a(x)} \int_{0}^{y} 1 d \theta=\frac{g(x)}{a(x)} y
$$

and we obtain that 


$$
\frac{a(x)}{g(x)} I I \rightarrow \epsilon y .
$$

Now consider the first term. By assumption we have

$$
\frac{a(x)}{g(x)}\left(\frac{U(x+y g(x))}{U(x)}-1\right) \rightarrow 0 .
$$

We conclude that

$$
\frac{a(x)}{g(x)}(I+I I) \rightarrow \epsilon y .
$$

Moreover, as we argued before, this relation holds l.u. in $y>0$. This implies that for $x$ large enough, the sign of $I+I I$ is the same as the sign of $\epsilon$. The inequalities of the theorem then follow.

Now we prove a converse result.

Starting from the inequalities in the theorem, for $y>0$ and $\epsilon>0$ we have

$$
\begin{aligned}
& \exp \epsilon \int_{x}^{x+y g(x)} a^{-1}(t) d t \\
& \quad \geq \frac{U(x+y g(x))}{U(x)} \geq \exp -\epsilon \int_{x}^{x+y g(x)} a^{-1}(t) d t
\end{aligned}
$$

with $a$ and $g$ as above. It follows that

$$
-\epsilon y \leq \lim \left(\begin{array}{c}
\sup \\
\inf
\end{array}\right) \frac{a(x)}{g(x)}\left(\frac{U(x+y g(x))}{U(x)}-1\right) \leq \epsilon y .
$$

Since $\epsilon$ was arbitrary, we find that

$$
\frac{a(x)}{g(x)}\left(\frac{U(x+y g(x))}{U(x)}-1\right) \rightarrow 0 .
$$

The theorem then follows.

Special Cases

(a) As a first case, we take $g(x)=1$ and $a(x)=x$, $x^{\circ}=1$. In this case we find back the result of Seneta as mentioned above.

(b) As a second case, we take $g(x)=1$ and $a(x)=$ $\sqrt{x}, x^{\circ}=0$. We find that

$$
\sqrt{x}\left(\frac{U(x+y)}{U(x)}-1\right) \rightarrow 0
$$

if and only if for $x$ large and $\epsilon>0$ we have

$$
\begin{gathered}
U(x+y) \times \exp \epsilon \int_{0}^{x+y} t^{-1 / 2} d t \\
\geq U(x) \times \exp \epsilon \int_{0}^{x} t^{-1 / 2} d t
\end{gathered}
$$

and

$$
\begin{array}{r}
U(x+y) \times \exp -\epsilon \int_{0}^{x+y} t^{-1 / 2} d t \\
\leq U(x) \times \exp -\epsilon \int_{0}^{x} t^{-1 / 2} d t
\end{array}
$$

or

$U(x+y) \times \exp 2 \epsilon(x+y)^{1 / 2} \geq U(x) \times \exp 2 \epsilon x^{1 / 2}$

and

$$
\begin{gathered}
U(x+y) \times \exp -2 \epsilon(x+y)^{1 / 2} \\
\leq U(x) \times \exp -2 \epsilon x^{1 / 2} .
\end{gathered}
$$

(c) Now we assume that $g(x)=1$ and $a(x) \in R V_{\lambda}$ with $0<\lambda<1$. In the case we find that

$$
\int_{x^{\circ}}^{x} \frac{1}{a(t)} d t \sim \frac{x}{a(x)(1-\lambda)},
$$

where $f(x) \sim g(x)$ means $\lim _{x \rightarrow \infty} f(x) / g(x)=$ 1. Using this relationship, we can alter the inequalities in the lemma to find

$$
U(x+y) \times \exp \epsilon A(x+y) \geq U(x) \times \exp \epsilon A(x)
$$

and

$$
\begin{gathered}
U(x+y) \times \exp -\epsilon A(x+y) \\
\leq U(x) \times \exp -\epsilon A(x),
\end{gathered}
$$

where $A(x)=x / a(x) \in R V_{1-\lambda}$.

(d) As a next example, we take $V(x)=U(x) \times$ $e^{-\tau x}$. Clearly we have

$$
\frac{U(x+y)}{U(x)}-e^{\tau y}=e^{\tau y}\left(\frac{V(x+y)}{V(x)}-1\right)
$$

and then,

$$
b(x)\left(\frac{U(x+y)}{U(x)}-e^{\tau y}\right) \rightarrow 0
$$

if and only if

$$
b(x)\left(\frac{V(x+y)}{V(x)}-1\right) \rightarrow 0,
$$

and the previous results can be applied.

2.4. A representation theorem for $Z(g, a)$. The following result is inspired by a result of Bojanic and Karamata [2] on the Zygmund class, see e.g. Theorem 1.5.5 in [1].

Theorem 2. Assume that $g \in S N, a \in \Gamma_{0}(g)$, $g(x)=o(a(x))$ and $g(x)>0$. Assume that $U \in$ $Z(g, a)$. Then, for some function $f$ and for $x \geq A$ for some $A>0$, 


$$
U(x)=\exp c+\int_{A}^{x} f(t) d t
$$

where $a(x) f(x) \rightarrow 0$.

Proof. Let $U \in Z(g, a)$. Then, by Theorem 1, the inequalities in such a theorem hold. Taking logarithms in such inequalities, denoting $V(x)=$ $\log U(x)$, gives for $x \geq x^{\circ}$ for some $x^{\circ}>0$ and $\epsilon>0$

$$
\begin{gathered}
V(x+y g(x))+\epsilon \int_{x^{\circ}}^{x+y g(x)} a^{-1}(t) d t \\
\geq V(x)+\epsilon \int_{x^{\circ}}^{x} a^{-1}(t) d t
\end{gathered}
$$

and

$$
\begin{aligned}
& V(x+y g(x))-\epsilon \int_{x^{\circ}}^{x+y g(x)} a^{-1}(t) d t \\
& \leq V(x)-\epsilon \int_{x^{\circ}}^{x} a^{-1}(t) d t
\end{aligned}
$$

Whence we have

$$
V(x+y g(x))-V(x) \geq-\epsilon \int_{x}^{x+y g(x)} a^{-1}(t) d t
$$

and

$$
V(x+y g(x))-V(x) \leq \epsilon \int_{x}^{x+y g(x)} a^{-1}(t) d t,
$$

and thus

$$
|V(x+y g(x))-V(x)| \leq \epsilon \int_{x}^{x+y g(x)} a^{-1}(t) d t .
$$

We then have, put $t=x+\theta g(x)$,

$$
|V(x+y g(x))-V(x)| \leq \epsilon \frac{g(x)}{a(x)} \int_{0}^{y} \frac{a(x)}{a(x+\theta g(x))} d \theta .
$$

Since $a \in \Gamma_{0}(g)$, we have for $x \geq x^{*}$ for some $x^{*} \geq x^{\circ}$ and for any $\theta \in[0, y]$

$$
\frac{a(x)}{a(x+\theta g(x))} \leq 1+\epsilon .
$$

Thus we have, as $x \rightarrow \infty$,

$$
|V(x+y g(x))-V(x)| \leq(1+\epsilon) \epsilon y \frac{g(x)}{a(x)} \rightarrow 0 .
$$

This convergence is uniform on $y \in[0, A)$ for any $A>0$. Moreover, $V$ is ultimately absolutely continuous, thus then $V$ is ultimately differentiable almost everywhere.

Therefore, we have, for $x>A$ for some $A \geq \max \left(x^{\circ}, x^{*}\right)$,

$$
V(x)=V(A)+\int_{A}^{x} f(t) d t
$$

where $f$ is a measurable function satisfying $f(x)=$ $V^{\prime}(x)$ almost everywhere in $(A, \infty)$. One may redefine $f(x)=0$ where $V^{\prime}(x)$ does not exist. Also, according to (5), we have, taking $z=x+y g(x)$, for $x \geq A$,

$$
a(x)|V(z)-V(x)| \leq(1+\epsilon) \epsilon|z-x| .
$$

Then, when $y \rightarrow 0$, which implies that $z \rightarrow x$, we have, for $x$ enough large such that $V^{\prime}(x)$ exists,

$$
-(1+\epsilon) \epsilon \leq a(x) V^{\prime}(x) \leq(1+\epsilon) \epsilon
$$

which implies that $a(x) V^{\prime}(x) \rightarrow 0$ as $x \rightarrow \infty$. Hence, we have

$$
U(x)=\exp V(x)=\exp V(A)+\int_{A}^{x} f(t) d t .
$$

\section{Remarks 3 .}

(a) It is easy to prove that the converse of Theorem 2 holds.

(b) If $L$ is slowly varying in the sense of Zygmund, this function thus has the canonical representation given by, see e.g. [15],

$$
L(x)=\exp c+\int_{B}^{x} \frac{\epsilon(t)}{t} d t,
$$

for a constant $c$ and a bounded measurable function $\epsilon(x)$ which satisfies $\epsilon(x) \rightarrow 0$ as $x \rightarrow$ $\infty$. Taking $g(x)=1$ and $a(x)=x$, these conditions satisfy $g \in S N$ and $g(x)=o(a(x))$. We then have $a(x)(\epsilon(x) / x) \rightarrow 0$ as $x \rightarrow \infty$. The previous remark thus implies that $L \in Z(g, a)$.

\section{References}

[ 1 ] N. H. Bingham, C. M. Goldie and J. L. Teugels, Regular variation, Encyclopedia of Mathematics and its Applications, 27, Cambridge University Press, Cambridge, 1987.

[ 2 ] R. Bojanic and J. Karamata, On slowly varying functions and asymptotic relations, in Math. Research Center Tech. Report, 432, Madison, Wisconsin, 1963.

[ 3 ] L. Y. Chan, Some properties of asymptotic functions and their applications, Proc. Amer. Math. Soc. 72 (1978), no. 2, 239-247.

[ 4 ] Y. Chen, Theorems of asymptotic approximation, Math. Ann. 140 (1960), 360-407.

[ 5 ] Y. Chen, On two-functional spaces, Studia Math. 24 (1964), 61-88.

[ 6 ] J. Karamata, Sur un mode de croissance régulière 
des fonctions, Mathematica (Cluj) 4 (1930), 3853 .

[ 7 ] J. Karamata, Sur un mode de croissance régulière. Théorèmes fondamentaux, Bull. Soc. Math. France 61 (1933), 55-62.

[ 8 ] R. Koenker and I. Mizera, Quasi-concave density estimation, Ann. Statist. 38 (2010), no. 5, 2998-3027.

[ 9 ] J. Németh, Generalizations of the Hardy-Littlewood inequality. II, Acta Sci. Math. (Szeged) 35 (1973), 127-134.

[ 10 ] E. Omey, On the class gamma and related classes of functions, Publ. Inst. Math. (Beograd) (N.S.) 93 (2013), no. 107, 1-18.

[11 ] L.-E. Persson, On a weak-type theorem with applications, Proc. London Math. Soc. (3) 38 (1979), no. 2, 295-308.

[ 12 ] L.-E. Persson and I. Wik, Integrability conditions on periodic functions related to their Fourier transforms, J. Math. Anal. Appl. 44 (1973), 291-309.

[13 G. Schechtman, Th. Schlumprecht and J. Zinn, On the Gaussian measure of the intersection, Ann. Probab. 26 (1998), no. 1, 346-357.

[14] E. Seneta, Regularly varying functions, Lecture Notes in Mathematics, Vol. 508, SpringerVerlag, Berlin, 1976.

[ 15 ] E. Seneta, Slowly varying functions in the Zygmund sense and generalized regular variation, J. Math. Anal. Appl. 475 (2019), no. 2, 16471657.

[16 ] A. Zygmund, On a theorem of Marcinkiewicz concerning interpolation of operations, J. Math. Pures Appl. (9) 35 (1956), 223-248.

[17] A. Zygmund, Trigonometric series. 2nd ed. Vols. I, II, Cambridge University Press, New York, 1959. 